\title{
HIV and SARS-CoV-2 Co-infection: Epidemiological, Clinical Features, and Future Implications for Clinical Care and Public Health for People Living with HIV (PLWH) and HIV Most-at-Risk Groups
}

\author{
Daniel K Nomah ${ }^{1,2,3}$ • Juliana Reyes-Urueña ${ }^{1,3,4}$. Josep Ma Llibre ${ }^{5}$ Juan Ambrosioni ${ }^{6} \cdot$ Fabiana S Ganem ${ }^{1,2,3}$. \\ José Ma Miró ${ }^{6}$. Jordi Casabona ${ }^{1,2,3,4}$
}

Accepted: 10 September 2021 / Published online: 10 December 2021

(c) The Author(s), under exclusive licence to Springer Science+Business Media, LLC, part of Springer Nature 2021

\begin{abstract}
Purpose of Review The purpose of this review is using the currently available clinical and epidemiological data, to identify key aspects to improve both the clinical management and public health response with regard SARS-CoV-2/HIV co-infection among HIV vulnerable populations and people living with HIV (PLWH).

Recent Findings While at the beginning of the COVID-19 pandemic, the lack of robust information on SARS-CoV-2/HIV coinfection prevented to have a clear picture of the synergies between them, currently available data strongly supports the importance of common structural factors on both the acquisition and clinical impact of these infections and the relevance of age, co-morbidities, and HIV viral load as associated worse prognosis factors among PLWH.

Summary Although more information is needed to better understand the biological, clinical, and epidemiological relationship between both infections, in the meanwhile, syndemic approaches to prevent SARS-CoV-2 among HIV higher risk groups and PLWH, targeting these population for SARS-CoV-2 vaccines and protocolizing early identification of HIV + patients with worse COVID-19 prognosis factors, are crucial strategies to decrease the overall impact of SARS-CoV-2/HIV coinfection.
\end{abstract}

Keywords HIV · AIDS · SARS-CoV-2 · COVID-19 · Coinfection · Epidemiology · Clinical guidelines · Impact · Synergia · Sindemia

This article is part of the Topical Collection Co-infections and Comorbidity

Jordi Casabona

jcasabona@iconcologia.net

1 Centre Estudis Epidemiològics Sobre Les Infeccions de Transmissió Sexual I Sida de Catalunya (CEEISCAT), Departament de Salut, Generalitat de Catalunya, Campus de Can Ruti, Josep Carreras Building, Ctra de Can Ruti, Camí de les Escoles, s/n, 08916 Badalona, Catalonia, Spain

2 Departament de Pediatria, d'Obstetrícia I Ginecologia I de Medicina Preventiva I de Salut Publica, Universitat Autònoma de Barcelona, Bellaterra, Spain

3 Institut d'Investigació Germans Trias I Pujol (IGTP), Badalona, Spain

4 CIBER Epidemiologia Y Salud Pública (CIBERESP), Barcelona, Spain

5 Hospital Universitari Germans Trias I Pujol, Badalona, Spain

6 Hospital Clínic-Institut d'Investigacions Biomèdiques August Pi I Sunyer, University of Barcelona, Barcelona, Spain

\section{Introduction}

As we make efforts to grapple with HIV, the novel COVID19 pandemic caused by the severe acute respiratory syndrome coronavirus 2 (SARS-CoV-2) has been an unprecedented threat to global health, challenging the robustness of health systems and the overall economy as a whole. Since the first case was reported in Wuhan, China, in 2019, infections are approaching 200 million and has claimed more than 4 million lives as of July 22, 2021 [1]. Older age and the presence of chronic co-morbidities like hypertension, diabetes, obesity, chronic respiratory disease, chronic kidney disease, cardiovascular disease, and malignancies are currently linked with a higher risk of severe disease and mortality [2-5]. On the other hand, as it happens with many other transmissible infections, COVID-19 vulnerability is also associated to social and structural determinants like poverty, population density, and lack of access to health services, creating a syndemia of social and health problems [6]. 
In this context, HIV most-at-risk populations share many structural factors for SARS-CoV-2 acquisition amidst the existing controversy regarding the susceptibility of people living with HIV (PLWH) to SARS-CoV-2 infection and its severity. PLWH have higher susceptibility to other opportunistic infections including respiratory infections due to their aberrant humoral and T-cell-meditated immune responses [7]. Moreover, with PLWH aging [8], having higher prevalence of chronic co-morbidities [9] and presenting with other risk factors of severe COVID-19 such as smoking [10], this population might have an increased risk to poorer COVID19 outcomes.

Although there are antecedents of two previous coronavirus epidemics, the severe acute respiratory syndrome (SARS) in 2003 and the Middle East Respiratory Syndrome (MERS) in 2013 [11], there is little to learn from these experiences regarding the incidence and clinical severity of HIV co-infection with these two coronaviruses. Current available data do not suggest a higher SARS-CoV-2 infection rates among PLWH [12-15], and although initial studies assessing the severity of COVID-19 among PLWH showed no clear evidence of poorer outcomes [16], emerging data are reporting higher risk of morbidity [13] and mortality [17-20] among some HIV-SARS-CoV-2 co-infected patients, particularly those with lower CD4 cell counts and those with an unsuppressed HIV viraemia [21-23]. Some researchers have also speculated a lower risk of infection and severe outcomes among HIV/SARS-CoV-2-co-infected patients because of the potential protection from some antiretroviral therapy (ART) regimens $[12,24,25]$ and have even suggested that immunosuppression could restrict the development of COVID-19-related cytokine storm [26, 27].

Finally, governments across the globe implemented strict measures to reduce mobility of persons and limit social activities in order to control the transmission of SARSCoV-2. These measures disrupted daily human lives and inadvertently hampered the delivery of key preventive and clinical HIV services [28].

The inconsistency of clinical data regarding the impact of SARS-CoV-2 on HIV and the scarcity of populationbased data sources which reports both infections highlight the importance of robust information systems with sociodemographic, biological, and clinical data to respond to the increasing number of questions needed to be answered to better articulate an effective response and to improve both clinical and public health guidelines on HIV-SARS-CoV-2 coinfections.

We have reviewed the association of HIV and SARSCoV-2 and its implications for HIV services from both a clinical and public health perspective, with a particular emphasis on the European context.

\section{Epidemiology of HIV and SARS-CoV-2}

Estimates from the UNAIDS show that as of the end of 2020, there were 37.6 million (estimate range, 30.2-45.0 million) people living with HIV with 1.5 million new cases and 690,000 deaths in 2020 only [29]. Since it was identified in 1980s in the World Health Organization (WHO) European Region, the HIV epidemic remains an on-going public health problem in the region with over 2 million people living with the infection by the end of 2019 [30]. In 2019 , there were 136,449 reported new cases in the region representing a crude rate of 15.6 per 100,000 persons of newly diagnosed infections [30]. Late diagnosis remains a challenge in the region as $31 \%$ of newly diagnosed persons presented with advanced HIV (CD4 $<200$ cells $/ \mathrm{mm}^{3}$ ) [30].

The COVID-19 pandemic first hit Europe on January 24, 2020, with the first three cases reported in France [31]. As of July 22, 2021, the region had reported 33,956,561 cases and 742,847 deaths [32]. France, UK, Italy, Spain, Germany, and Poland have been hugely affected in the European region in terms of number of confirmed cases and deaths [32]. The European monitoring of excess mortality for public health action (EuroMOMO) network reported excess mortality estimates in the initial stages of the COVID-19 pandemic in Europe. By the 18th week after the pandemic commenced in Europe, the cumulative excess mortality in all ages was 185,287 deaths. People aged $\geq 85$ years contributed most to the excess mortality (48\%) and was least among in 15-44-year-olds (1\%) [33].

Across Europe, the incidence of COVID-19 among PLWH ranges from 0.3 to $5.7 \%$ person years [12, 14, 22, $34,35]$. Two studies have assessed the prevalence of HIV among hospitalized COVID-19 patients in Europe being between 0.26 and $1.0 \%[20,36]$. In a pooled analysis, PLWH were identified to be more susceptible to SARSCoV-2 infection with a risk ratio (RR) of 1.24 (95\% confidence interval [95\% CI] 1.05-1.46); the prevalence of HIV among hospitalized COVID-19 patients in this analysis was however not significantly different than the background population ( $\mathrm{RR}=1.22,95 \% \mathrm{CI} 0.61-2.65)$ [37]. Nevertheless, because diagnostic rates are a function of the screening criteria, it is likely that-particularly at the beginning of the pandemic_-diagnostic rates among PLWH were overestimated because testing was based on symptoms, age, co-morbidities, or epidemiological criteria.

HIV/SARS-CoV-2-co-infected patients in Europe were 38-56 years which is about a decade younger than the reported average age in the general population [20] and predominantly males [12, 14, 20, 22, 34, 35]. COVID19-diagnosed PLWH were more likely to belong to a low socioeconomic class [17] and likely to be of black 
ethnicity $[17,20]$. Mortality rates among HIV/SARS-CoV2-coinfected patients in Europe have ranged from 1.9 to $29.0 \%$ [12, 14, 20, 21, 34-36, 38-42].

An essential aspect of any infectious disease control and prevention measure is quality epidemiological surveillance, timely and accurate enough to identify cases and patterns of transmission to guide early and effective preventive interventions. Both in the USA [43] and Europe [44], retrospective analysis of influenza monitoring sentinel networks suggested that SARS-CoV-2 was circulating earlier than the first cases officially recognized and therefore a high percentage of these infections remaining undiagnosed. A study from Wuhan, China, reported that if public health measures had begun 3 weeks earlier, cases could have been reduced by $95 \%$ [45].

Robust population-based programmatic and surveillance data, including epidemiological, microbiological, clinical, and syndromic information, are crucial to understand the distribution and dynamics of SARS-CoV-2 and its impact in key populations such as PLWH. Unfortunately, at the beginning of the COVID-19 pandemic, many national information systems were not prepared to face an integrated approach of the needs emerged by the SARS-CoV-2 infection. Systematizing the use of microbiological methods to identify SARS-CoV-2 infection among PLWH is imperative not only to have a better picture of the epidemiological pattern of the co-infection, but also to identify individuals at risk of worse clinical outcomes promptly.

\section{Potential Impact of Antiretrovirals on SARS-CoV-2 Infection Risk and Outcomes}

Since the beginning of the COVID-19 pandemic, the possibility that some antiretroviral drugs might be active against SARS-CoV2 was considered. However, with the evidence available so far, the antiretroviral treatment should not be modified to treat SARS-CoV-2, since no proven activity of any antiretroviral drug has been consistently documented. Some studies are ongoing to bring light to this issue. The association of tenofovir disoproxil (TDF) use and a potentially lower COVID-19 infection rate has sparked much debate. In studies from Spain [12] and South-Africa [18], TDF was associated with lower SARS-CoV2 infection incidence rates.

However, selection biases were present in both studies since it is unlikely to provide TDF to PLWH with known co-morbidities such as cardiovascular risk factors or kidney disease, which have been identified as risk factors for COVID-19 and poor outcomes. Therefore, PLWH treated with TDF would probably be preferentially younger and with no known comorbidities, entailing an intrinsic lower risk for SARS-CoV-2 infection. In the South-African study, receipt of TDF (vs. zidovudine) was associated with reduced COVID-19 mortality even after adjusting for renal disease, viral suppression, and antiretroviral treatment duration, but again, other non-adjusted factors (prior virologic failure, tuberculosis) could be associated with zidovudine use [18]. A third study in subjects with chronic hepatitis B (without HIV infection) has also reported significantly lower rates of severe COVID-19, intensive care unit (ICU) admission, ventilatory support, and fewer days in the hospital in those receiving TDF vs. entecavir [46]. However, again, people taking TDF had significantly lower co-morbidity rates. Finally, TDF/FTC PrEP users presented a higher seroprevalence to SARS-CoV-2 than the control group, with no significant differences in clinical manifestations [47]. Similarly, a matched case-control comparison in the PREVENIRANRS French study with middle-aged HIV-negative people who did or did not use TDF/FTC PrEP found no evidence that TDF may help ward off SARS-CoV-2 infection [48].

HIV protease-inhibitors (mainly lopinavir/ritonavir) have been used early during the pandemic to treat COVID-19 but, currently, no study supports its use.

\section{Potential Impact of Co-morbidities on SARS-CoV-2 Infection}

Although some studies have reported HIV infection as a risk factor for poor outcome, there is no clear evidence for a more severe disease course of SARS-CoV-2 infection in PLWH on active ART and with good cellular immunity (CD4 cell count) levels. Among hospitalized COVID-19 patients, most studies reported a younger age of PLWH vs. HIV-negative patients, with higher rates of comorbidity [14, 23, 35]. These co-morbidities are consistently associated with poorer outcomes in PLWH with well-controlled HIV infection and constitute the underlying bias in all series reported.

On the other hand, larger cohort studies suggested poorer outcomes for PLWH not only in the presence of co-morbidities, but also in those with low CD4 cell count $(<200$ cells/ $\mathrm{mm}^{3}$ ) $[18,23]$. This would be consistent with the poorer COVID-19 outcomes seen in other immunocompromised hosts. In addition, severe COVID-19 has been also described in PLWH with concomitant opportunistic infections, such as tuberculosis and/or Pneumocystis jirovecii [49].

Thus, PLWH have a higher risk for severe COVID-19 outcomes in two clinical contexts: (i) uncontrolled HIVinfection and/or advanced immunodeficiency, or (ii) additional co-morbidities.

\section{Outcomes (Hospitalization, Intensive Care Unit, and Mortality) and Determinants}

In studies including outpatients, overall rates of ICU admission ranged between 3 and 22\% for PLWH [50]. When only hospitalizations were reported, ICU admission for PLWH 
ranged between 17 and 33\% [50]. Severity of COVID-19 illness increases with age and comorbidities, as it does in the general population. Multimorbidities have been reported in nearly two-thirds of patients co-infected with HIV and SARS-CoV-2 [23, 50].

Despite the existing debate, clinical data from 37 countries reported to the WHO Global Clinical Platform for COVID-19 indicate that HIV infection is a significant independent risk factor for severe/critical COVID-19 presentation at hospital admission and in-hospital mortality [19]. Mortality in hospitalized PLWH with COVID in the UK showed an adjusted hazard ratio (aHR) of 1.69 (95\% CI $1.15-2.48 ; p=0.008$ ) [20], whereas, in primary care alone, after adjustment for age, gender, ethnicity, smoking, and obesity among other variables, the aHR was 2.59 $(1.74-3.84 ; p<0.0001)$. Most deceased PLWH had other co-morbidities [17]. Similar results were found in Western Cape, South Africa, in multivariate analysis with PLWH having a risk of death with COVID-19 of 2.14 (1.70-2.70) [18]. However, global mortality varied considerably across studies, depending on the design. Mortality was as high as $24 \%$ in the UK series (only hospitalizations) [20], as low as 2\% in a series from France [41] and 3.6\% in the South African cohort study [18] with a higher number of patients and the inclusion of outpatients, but also a more severely immunosuppressed population.

A recent analysis from the PISCIS Cohort Study in Catalonia (Spain) identified HIV co-infected patients with detectable HIV viral load, lower CD4 cell counts, older age, chronic comorbidities, and migrants as those with higher risks of severe outcomes [22]. Of note, lower CD4 cell counts were a risk factor to severe COVID-19 only among patients with detectable viral load in stratified analysis.

\section{European Guidelines on Clinical Management}

Clinical and radiological COVID-19 presentation is no different in PLWH to typical reports in the general population $[23,50]$.

The European AIDS Clinical Society and several National HIV/AIDS Societies have provided general recommendations for the management of PLWH with COVID-19 [51]. Essentially, the diagnostic approach and overall management should follow those indicated for the general population. Differential diagnosis should include other respiratory diseases such as Pneumocystis jirovecii and tuberculosis, particularly in severely immunocompromised PLWH.

However, when treating COVID-19 in PLWH, specific issues must be addressed. The first is to consider and check for drug-drug interactions between COVID-19 treatments and ARV drugs. The second is to consider the potential overlapped toxicities of ARV and SARS-CoV-2 therapies, such as liver or kidney toxicity of remdesivir with specific ARV drugs.

For PLWH with severe immune depletion and other types of immunosuppressed individuals (solid transplant recipients, onco-hematologic patients), prolonged SARS-CoV-2 replication and shedding have been reported, which may require longer isolation periods. However, no specific recommendation for testing and isolation has been provided yet for PLWH.

Finally, emerging data is showing that long COVID will have a great impact in the burden of disease both in developed and low- and middle-income countries (LMIC) [52]. Given the huge symptomatic spectrum and the lack of knowledge about its pathophysiological mechanism, PLWH exposed to SARS-CoV-2 should be monitored to clarify if they are at higher risk of experiencing some features of long COVID syndrome.

\section{SARS-CoV-2 Vaccination Among PLWH}

There is limited information of the immunogenicity and reactogenicity of commercially available COVID-19 vaccines in patients living with HIV (PLHIV). Studies analyzing the response of PLHIV to COVID-19 vaccines are needed to define the potential advantages and disadvantages of some types of vaccines (adenovirus-based vs. mRNA vs. recombinant spike vaccines) or the need for additional vaccine doses to achieve full protective immunity in immunosuppressed HIV-infected patients. The latter is an important point because it is well known that the immune damage to B-cell compartments and antibody generation caused by HIV infection can decrease the humoral response as it has been demonstrated to other vaccines (e.g., influenza or pneumococcal vaccines) [53, 54]. Therefore, dysfunctional B-cell memory lymphocytes and follicular helper T-cell activity could potentially decrease humoral responses to neo-antigens such as SARS-CoV-2 protein spike.

Data on PLWH included in approved phase $2 / 3$ vaccine trials so far is limited. Regarding mRNA vaccines, in the Moderna and Pfizer trials [55, 56], only $0.6 \%$ and $0.5 \%$ of participants were PLWH, respectively. The HIV sub-study results of these trials have not been published yet. However, in another small study in 12 PLWH (seven women, five men), the Pfizer vaccine induced, between 7 and 17 days after the second vaccine dose, a robust humoral and cellular immune responses comparable to that seen in 17 healthy donors (seven women, 10 men) [57]. Regarding the adenovirus-based vaccines, the safety and immunogenicity of the ChAdOx1 nCoV-19 (AZD1222) vaccine [58] were evaluated in 54 individuals in a single-arm open-label vaccination substudy. All participants were men (most white) on ART, with 
undetectable plasma HIV viral loads $(<50$ copies $/ \mathrm{mL})$, and CD4 counts of more than 350 cells $/ \mathrm{mm}^{3}$ (median 700 cells/ $\mathrm{mm}^{3}$ ). No serious adverse events occurred. Anti-spike IgG responses peaked at day 42 and were sustained until day 56 with no correlation with CD4 cell count or age. Compared with HIV-negative participants, the study found no differences in magnitude or persistence of SARS-CoV-2 spike-specific humoral or cellular responses. Finally, using the recombinant spike protein, the NVX-CoV2373 vaccine phase $2 \mathrm{~b}$ trial [59] performed in South Africa included 6\% of PLWH. The vaccine efficacy in PLWH was lower than in non-HIV-individuals (52 vs. $60 \%$ ).

Immunosuppressed PLWH should be considered a risk group in which early COVID-19 vaccination would be advised as supported by European AIDS Clinical Society (EACS) Guidelines [51]. PLWH with low CD4 counts (below 200 cells $/ \mu \mathrm{L}$ or even below 350 cells $/ \mu \mathrm{L}$ ) are at higher risk of developing severe COVID-19 [22] and could be considered as a priority group for COVID-19 vaccination. Therefore, we urgently need studies that analyze the efficacy and safety of vaccines in these immunosuppressed patients, in patients without ART, in women, and in different races. In addition, we should also know the duration of the immune response since some PLWH may need additional doses of vaccines.

Finally, since there is evidence that HIV adenovirusbased vaccines, trials increased the risk of acquiring HIV [60-62], further information is also needed to better understand the appropriateness of using the adenovirus type 5 vectored vaccines among HIV most-at-risk groups.

\section{Impact of SARS-CoV-2 Pandemic in HIV Most-at-Risk-Groups and PLWH}

Aside the direct risks to physical health, the psychological impact of COVID-19 could also be detrimental to mental well-being as elevated levels of stress and anxiety are further exacerbated by the ongoing uncertainty of the situation [63]. Key populations among PLWH experience particular forms of exclusion, criminalization, inequality, and discrimination that render them particularly vulnerable to COVID-19 [64]. This burden can affect the physical, emotional, and social well-being of PLWH and interfere with the reception of effective healthcare and access to HIV treatment [65].

A survey of older PLWH in Miami, USA, found that participants reported increased stress associated with their sense of social isolation and fragile economic situation [66]. PLWH who reported higher levels of anxiety and depression also reported losing their jobs during the pandemic [67]. Another study has described that psychological stress might be a predictor of COVID-19 burden (financial and social burden) and COVID-19 risk (health factors associated with an increased risk of severe health outcomes due to COVID19) [67]. Additionally, COVID-19 burden and COVID19 risk were predictors of depression and sleep problems [68]. LGBT people reported an elevated risk of domestic and family violence, increased social isolation, difficulties in accessing crucial HIV treatment, and gender-affirming health services [67, 69].

The impact of COVID-19 on sexual behavior among gay and bisexual men living with HIV has been described in three studies which reported changes in sexual behaviors, including avoiding close physical contact and reducing or ceasing sex with casual partners $[70,71]$. There is also a reported increase in recreational drugs such as marijuana and methamphetamine (up to $8 \%$ ), and alcohol consumption and binge drinking [70-72].

\section{Impact on Health Services, Access to Diagnosis, and Treatment}

In addition to the health emergency caused by the COVID19, the pandemic has threatened the excellence in ART delivery in well-resourced countries which could potentially result in reduction in adherence and decreased health care retention [73, 74]. It is estimated that a significant proportion of HIV-infected patients could not access usual care because many HIV/AIDS prevention and control centers around the world have been converted into COVID-19 treatment centers and the perceived fear of contracting COVID-19 has made this group situation more vulnerable [75].

Vital HIV care resources including healthcare personnel have been channeled into curbing the COVID-19 pandemic [76]. A high percentage of community-based testing services have stopped or dramatically decreased their activity [77] and many HIV care centers worldwide were repurposed for the fight against COVID-19 denying PLWH the possibility of accessing crucial ART. [75]

During these periods, drug guarantee and distribution strategies were adopted by several countries; however, there are still uncertainties regarding the situation of assistance to PLWH in countries where the economy was highly affected. A study evaluating the impact of the pandemic on care for this population as well as on the provision of treatment found that no country, among the 19 participants, reported the closure of HIV care services; however, the functioning was normal in 6 countries (31.6\%) and in 11 of them (57.9\%) care was shared between HIV and COVID-19. Furthermore, the rechanneling of health professionals, especially HIV specialists, to the COVID-19 response caused the exhaustion of many HIV care teams [77].

For this reason, recommendations for management and prevention of COVID-19 among PLWH emphasize the need for continuity of HIV care, including uninterrupted access to 
ART, routine vaccinations, and the use of telematic means to access care, as long as it follows the general COVID-19 preventive guidelines. Also, continuous monitoring of the impact of the pandemic on this population is encouraged, so that it is possible to systematize evidence to support the reorganization of assisting services for PLWH. Regarding prevention services, the pandemic has challenged the functioning of HIV prevention services, such as free access to condoms and HIV pre-exposure prophylaxis (PrEP) and calls for innovative approaches going forward [78].

In Europe, some HIV prevention and diagnosis services have incorporated self-sampling and self-testing approaches [77]; nevertheless, applicability of these strategies may not be easy as they require good information technology and mailing systems, which is not the case in many LMIC. On the other hand, steady access to clinical care may be facilitated by means of telemedicine. But again, in light of the COVID-19 pandemic, as it has been described from an experience in the USA that it is likely that economic, geographic inequities, and the digital divide will prevent some PLWH from accessing care via this route due to lack of necessary technology (e.g., computer, smartphones) or stable internet access, especially among older PLWH [79].

\section{Conclusions}

In this review, we have highlighted some aspects of the SARS-CoV-2/HIV co-infection from both a public health and clinical perspective. Although at the beginning of the COVID-19 pandemic, the lack of integrated epidemiological surveillance and programmatic data prevented to have a clear picture of the overlapping distribution of both infections and its determinants, from a public health perspective, currently available data confirms the role of common structural determinants in both their acquisitions and clinical impact. Public health weakness, drug use, mental health, stigma, social marginalization, and other structural determinants they all disproportionally increase the exposure to both HIV and SARS-CoV-2.

From a clinical perspective, data suggest that PLWH under effective ARV treatment are not at higher risk of acquiring SARS-CoV-2. Moreover, there is no current evidence suggesting that TDF could reduce the risk of SARSCoV-2 infection or severe COVID-19 outcomes in PLWH, and both clinical and radiological features of COVID-19 in PLWH are similar to those without HIV infection. Nevertheless, HIV-associated co-morbidities, low CD4 ( $<200$ cells) cell counts, and in particular unsuppressed HIV viraemia are associated with poorer COVID-19 clinical outcomes and death among PLWH. In such a context, PLWH should be considered a priority target group for SARS-CoV-2 vaccinations and further information is needed to identify potential advantages or disadvantages of the different commercially available vaccines and vaccination schedules to be used into this population.

The SARS-CoV-2 pandemic has not only impacted PLWH, particularly disrupting access to ARV treatments, but also HIV most-at-risk groups-including men who have sex with men (MSM), sexual workers (SW), and people using drugs (PUD)_-decreasing access to HIV prevention and early diagnosis and linkage programs. Under the current uncertain epidemiological scenario, both clinical services offering medical care to PLWH and community programs offering services to vulnerable groups should be adapted to the evolving COVID-19 pandemic and the corresponding mitigation scenarios to prevent disruption of the HIV continuum of care among these populations. Systematization of SARS-CoV-2 testing among PLWH is basic not only to better understand the epidemiological co-infection pattern, but also to identify PLWH co-infected with SARS-CoV-2 with worse prognostic factors, as soon as possible. Integration of SARS-CoV-2 testing strategies in HIV testing programs should therefore be considered. With this regard, alternative approaches like telemedicine, self-sampling, and self-testing technologies have been used in both developed and LMIC with promising results and they should be scaled up.

Integrated information systems including epidemiological, microbiological, clinical, vaccination, and mortality data should be reinforced and maintained to monitor the impact of COVID among PLWH and HIV most-at-risk-groups. The use of PLWH longitudinal clinical data, populationbased SARS-CoV-2 diagnosis data as well as ecological approaches to include structural indicators, seems crucial to increase the knowledge and practices towards SARSCoV-2/HIV coinfection from both a clinical and public health perspective.

Funding JMM received a personal 80:20 research grant from Institut d'Investigacions Biomèdiques August Pi i Sunyer (IDIBAPS), Barcelona, Spain, during 2017-21.

\section{Declarations}

Conflict of Interest JMM has received consulting honoraria and/or research grants from AbbVie, Angelini, Contrafect, Cubist, Genentech, Gilead Sciences, Jansen, Lysovant, Medtronic, MSD, Novartis, Pfizer, and ViiV Healthcare, outside the submitted work. All other authors, no conflicts.

\section{References}

1. WHO Coronavirus Disease (COVID-19) Dashboard. In: Geneva World Heal. Organ. https://covid19.who.int/. Accessed 21 May 2020 
2. Richardson S, Hirsch JS, Narasimhan M, et al. Presenting characteristics, comorbidities, and outcomes among 5700 patients hospitalized with COVID-19 in the New York City area. JAMA. 2020;323:2052-9.

3. Onder G, Rezza G, Brusaferro S. Case-fatality rate and characteristics of patients dying in relation to COVID-19 in Italy. JAMA. 2020;323:1775-6.

4. Zhou F, Yu T, Du R, Fan G, Liu Y, Liu Z, Xiang J, Wang Y, Song B, Gu X. Clinical course and risk factors for mortality of adult inpatients with COVID-19 in Wuhan, China: a retrospective cohort study. Lancet. 2020;395:1054-62.

5. Wu Z, McGoogan JM. Characteristics of and important lessons from the coronavirus disease 2019 (COVID-19) outbreak in China: summary of a report of 72314 cases from the Chinese Center for Disease Control and Prevention. JAMA. 2020;323:1239-42.

6. Sachs JD, Horton R, Bagenal J, Ben AY, Caman OK, Lafortune G. The lancet COVID-19 commission. Lancet. 2020;396:454-5.

7. Chang CC, Crane M, Zhou J, Mina M, Post JJ, Cameron BA, Lloyd AR, Jaworowski A, French MA, Lewin SR. HIV and coinfections. Immunol Rev. 2013;254:114-42.

8. Smit M, Brinkman K, Geerlings S, Smit C, Thyagarajan K, van Sighem A, de Wolf F, Hallett TB. Future challenges for clinical care of an ageing population infected with HIV: a modelling study. Lancet Infect Dis. 2015;15:810-8.

9. Guaraldi G, Orlando G, Zona S, Menozzi M, Carli F, Garlassi E, Berti A, Rossi E, Roverato A, Palella F. Premature age-related comorbidities among HIV-infected persons compared with the general population. Clin Infect Dis. 2011;53:1120-6.

10. Tesoriero JM, Gieryic SM, Carrascal A, Lavigne HE. Smoking among HIV positive New Yorkers: prevalence, frequency, and opportunities for cessation. AIDS Behav. 2010;14:824-35.

11. Peeri NC, Shrestha N, Rahman MS, Zaki R, Tan Z, Bibi S, Baghbanzadeh M, Aghamohammadi N, Zhang W, Haque U. The SARS, MERS and novel coronavirus (COVID-19) epidemics, the newest and biggest global health threats: what lessons have we learned? Int J Epidemiol. 2020;49:717-26.

12. del Amo J, Polo R, Moreno S, Díaz A, Martínez E, Arribas JR, Jarrín I, Hernán MA. Incidence and severity of COVID-19 in HIV-positive persons receiving antiretroviral therapy. Ann Intern Med. 2020. https://doi.org/10.7326/M20-3689.

13. Tesoriero JM, Swain C-AE, Pierce JL, et al. COVID-19 outcomes among persons living with or without diagnosed HIV infection in New York State. JAMA Netw Open. 2021;4:e2037069-e2037069.

14. Vizcarra P, Pérez-Elías MJMJMJ, Quereda C, et al. Description of COVID-19 in HIV-infected individuals: a single-centre, prospective cohort. Lancet HIV. 2020;3018:1-11.

15. Huang J, Xie N, Hu X, et al. Epidemiological, virological and serological features of coronavirus disease 2019 (COVID-19) cases in people living with human immunodeficiency virus in Wuhan: a population-based cohort study. Clin Infect Dis. 2020. https://doi.org/10.1093/cid/ciaa1186.

16. Cooper T, Woodward B, Alom S, Harky A. Coronavirus disease 2019 (COVID-19) outcomes in HIV/AIDS patients: a systematic review. HIV Med. 2020;21:567-77.

17. Bhaskaran K, Rentsch CT, MacKenna B, et al. HIV infection and COVID-19 death: a population-based cohort analysis of UK primary care data and linked national death registrations within the OpenSAFELY platform. Lancet HIV. 2021;8:e24-32.

18. Boulle A, Davies M-A, Hussey H, et al. Risk factors for coronavirus disease 2019 (COVID-19) death in a population cohort study from the Western Cape Province South Africa. Clin Infect Dis. 2020. https://doi.org/10.1093/cid/ciaa1198.

19. Bertagnolio S, Thwin S, Silva R, Ford N, Baggaley R, Vitoria M, Jassat W, Doherty M Clinical characteristics and prognostic factors in people living with HIV hospitalized with COVID-19: findings from the WHO Global Clinical Platform. 11th IAS Conf. HIV Sci. I 18-21 July 2021. PEBLB20

20. Geretti AM, Stockdale AJ, Kelly SH, et al. Outcomes of coronavirus disease 2019 (COVID-19) related hospitalization among people with human immunodeficiency virus (HIV) in the ISARIC World Health Organization (WHO) Clinical Characterization Protocol (UK): a prospective observational study. Clin Infect Dis. 2020. https://doi.org/10.1093/cid/ciaa1605.

21. Hoffmann C, Casado JL, Härter G, et al (2020) Immune deficiency is a risk factor for severe COVID-19 in people living with HIV. HIV Med. 1-7

22. Nomah DK, Reyes-Urueña J, Díaz Y, Moreno S, Aceiton J, Bruguera A, Vivanco-Hidalgo RM, Llibre JM, Domingo P, Falco V, Imaz A, Cortes C, Force L, Letang E, Vilaro I, Casabona J, Miro JM; PISCIS study group. Sociodemographic, clinical, and immunological factors associated with SARS-CoV-2 diagnosis and severe COVID-19 outcomes in people living with HIV: a retrospective cohort study. Lancet HIV. 2021;8(11):e701-10. https:// doi.org/10.1016/S2352-3018(21)00240-X

23. Dandachi D, Geiger G, Montgomery MW, et al. Characteristics, comorbidities, and outcomes in a multicenter registry of patients with human immunodeficiency virus and coronavirus disease 2019. Clin Infect Dis. 2020;65212:1-9.

24. Cao B, Wang Y, Wen D, et al. A trial of lopinavir-ritonavir in adults hospitalized with severe covid-19. N Engl J Med. 2020;382:1787-99.

25. Zanella I, Zizioli D, Castelli F, Quiros-Roldan E. Tenofovir, another inexpensive, well-known and widely available old drug repurposed for SARS-COV-2 infection. Pharmaceuticals. 2021;14:454.

26. Guo W, Ming F, Dong Y, et al. A survey for COVID-19 among HIV/AIDS patients in two Districts of Wuhan, China. Lancet. 2020. https://doi.org/10.2139/ssrn.3550029.

27. Shalev N, Scherer M, LaSota ED, Antoniou P, Yin MT, Zucker J, Sobieszczyk ME. Clinical characteristics and outcomes in people living with HIV hospitalized for COVID-19. Clin Infect Dis an Off Publ Infect Dis Soc Am. 2020. https://doi.org/10.1093/cid/ ciaa635.

28. Sun S, Hou J, Chen Y, Lu Y, Brown L, Operario D. Challenges to HIV care and psychological health during the COVID-19 pandemic among people living with HIV in China. AIDS Behav. 2020;24:2764-5.

29. Joint United Nations Programme on HIV/AIDS (UNAIDS). (2021) FACT SHEET 2021. Preliminary UNAIDS 2021 epidemiological estimates. GLOBAL HIV STATISTICS.

30. European Centre for Disease Prevention and Control/WHO Regional Office for Europe. (2020) HIV/AIDS surveillance in Europe 2020. 2019 data.

31. Stoecklin SB, Rolland P, Silue Y, Mailles A, Campese C, Simondon A, Mechain M, Meurice L, Nguyen M, Bassi C. First cases of coronavirus disease 2019 (COVID-19) in France: surveillance, investigations and control measures, January 2020. Eurosurveillance. 2020;25:2000094.

32. European Centre for Disease Prevention and Control (2021) COVID-19 situation update for the EU/EEA, as of 1 July 2021. https://www.ecdc.europa.eu/en/cases-2019-ncov-eueea. Accessed $22 \mathrm{Jul} 2021$

33. Vestergaard LS, Nielsen J, Richter L, Schmid D, Bustos N, Braeye T, Denissov G, Veideman T, Luomala O, Möttönen T. Excess allcause mortality during the COVID-19 pandemic in Europe-preliminary pooled estimates from the EuroMOMO network, March to April 2020. Eurosurveillance. 2020;25:2001214.

34. Gervasoni C, Meraviglia P, Riva A, Giacomelli A, Oreni L, Minisci D, Atzori C, Ridolfo A, Cattaneo D (2020) Clinical features and outcomes of HIV patients with coronavirus disease 2019. Clin Infect Dis ciaa579 
35. Inciarte A, Gonzalez-Cordon A, Rojas J, et al. Clinical characteristics, risk factors, and incidence of symptomatic coronavirus disease 2019 in a large cohort of adults living with HIV: a single-center, prospective observational study. AIDS. 2020;34:1775-80.

36. Blanco JL, Ambrosioni J, Garcia F, Martínez E, Soriano A, Mallolas J, Miro JM. COVID-19 in patients with HIV: clinical case series. Lancet HIV. 2020;7:e314-6.

37. Ssentongo P, Heilbrunn ES, Ssentongo AE, Advani S, Chinchilli VM, Nunez JJ, Du P. Epidemiology and outcomes of COVID19 in HIV-infected individuals: a systematic review and metaanalysis. Sci Rep. 2021;11:1-12.

38. Cabello A, Zamarro B, Nistal S, Victor V, Hernández J, PrietoPérez L, Carrillo I, Álvarez B, Fernández-Roblas R, HernándezSegurado M. COVID-19 in people living with HIV: a multicenter case-series study. Int J Infect Dis. 2021;102:310-5.

39. Kowalska JD, Kase K, Vassilenko A, Harxhi A, Lakatos B, Lukić GD, Verhaz A, Yancheva N, Dumitrescu F, Jilich D. The characteristics of HIV-positive patients with mild/asymptomatic and moderate/severe course of COVID-19 diseasea report from Central and Eastern Europe. Int J Infect Dis. 2021;104:293-6.

40. Di Biagio A, Ricci E, Calza L, Squillace N, Menzaghi B, Rusconi S, Orofino G, Bargiacchi O, Molteni C, Valsecchi L. Factors associated with hospital admission for COVID-19 in HIV patients. AIDS. 2020;34:1983-5.

41. Etienne N, Karmochkine M, Slama L, Pavie J, Batisse D, Usubillaga R, Letembet V-A, Brazille P, Canouï E, Slama D. HIV infection and COVID-19: risk factors for severe disease. AIDS. 2020;34:1771.

42. Childs K, Post FA, Norcross C, Ottaway Z, Hamlyn E, Quinn K, Juniper T, Taylor C (2020) Hospitalized patients with COVID-19 and human immunodeficiency virus: a case series. Clin Infect Dis $1-2$

43. Silverman JD, Hupert N, Washburne AD (2020) Using influenza surveillance networks to estimate state-specific prevalence of SARS-CoV-2 in the United States. Sci. Transl. Med. 12:

44. Coma E, Mora N, Prats-Uribe A, Fina F, Prieto-Alhambra D, Medina-Peralta M (2020) Excess cases of influenza suggest an earlier start to the coronavirus epidemic in Spain than official figures tell us: an analysis of primary care electronic medical records from over 6 million people from Catalonia. Medrxiv

45. Peixoto VR, Nunes C, Abrantes A. Epidemic surveillance of Covid-19: considering uncertainty and under-ascertainment. Port J Public Heal. 2020;38:23-9.

46. Muñoz B, Buti M, VAzquez I Tenofovir reduces severity of COVID-19 infection in chronic hepatitis B patients. EASL Int. Liver Congr. June 23-26, 2021. Abstr. PO-1449.

47. Ayerdi O, Puerta T, Clavo P, Vera M, Ballesteros J, Fuentes ME, Estrada V, Rodríguez C, Del Romero J. Preventive efficacy of tenofovir/emtricitabine against severe acute respiratory syndrome coronavirus 2 among pre-exposure prophylaxis users. Open Forum Infect Dis. 2020. https://doi.org/10.1093/ofid/ofaa455.

48. Delaugerre C, Assoumou L, Maylin S SARS CoV-2 seroprevalence among HIV-negative participants using tenofovir/emtricitabine-based PrEP in 2020: a sub-study of PREVENIR-ANRS and SAPRIS-Sero. IAS 2021, 11th IAS Conf. HIV Sci. July 18-21, 2021. Abstr. OAC0201.

49. Feldman C, Anderson R. The role of co-infections and secondary infections in patients with COVID-19. Pneumonia. 2021;13:5.

50. Ambrosioni J, Blanco JL, Reyes-urueña JM, Davies M, Sued O, Marcos MA, Martínez E. Overview of SARS-CoV-2 infection in adults living with HIV. Lancet HIV. 2021;8:294-305.

51. European AIDS Clinical Society (EACS) (2021) Statement on risk of COVID-19 for people living with HIV (PLWH) and SARSCoV-2 vaccine advice for adults living with HIV. https://www. eacsociety.org/home/bhiva-daig-eacs-gesida-polish-scientificaids-society-and-portuguese-association-for-the-clinical-study-ofaids-apecs-statement-on-risk-of-covid-19-for-people-living-withhiv-plwh-and-sars-cov-2-vaccine-advice-for-adults-li. Accessed 21 Jul 2021

52. World Health Organization (WHO) (2021) Expanding our understanding of post COVID-19 condition: report of a WHO webinar, 9 February 2021.

53. Lee K-Y, Tsai M-S, Kuo K-C, Tsai J-C, Sun H-Y, Cheng AC, Chang S-Y, Lee C-H, Hung C-C. Pneumococcal vaccination among HIV-infected adult patients in the era of combination antiretroviral therapy. Hum Vaccin Immunother. 2014;10:3700-10.

54. Ceravolo A, Orsi A, Parodi V, Rosselli R, Ansaldi F. Influenza vaccination in HIV-positive subjects: latest evidence and future perspective. J Prev Med Hyg. 2013;54:1.

55. Polack FP, Thomas SJ, Kitchin N, Absalon J, Gurtman A, Lockhart S, Perez JL, Pérez Marc G, Moreira ED, Zerbini C. Safety and efficacy of the BNT162b2 mRNA Covid-19 vaccine. N Engl J Med. 2020;383:2603-15.

56. Baden LR, El Sahly HM, Essink B, Kotloff K, Frey S, Novak R, Diemert D, Spector SA, Rouphael N, Creech CB (2020) Efficacy and safety of the mRNA-1273 SARS-CoV-2 vaccine. N Engl J Med.

57. Woldemeskel BA, Karaba AH, Garliss CC, Beck EJ, Wang KH, Laeyendecker O, et al. The BNT162b2 mRNA vaccine elicits robust humoral and cellular immune responses in people living with HIV. Clin Infect Dis. 2021;22:ciab648. https://doi.org/10. 1093/cid/ciab648 (Online ahead of print).

58. Frater J, Ewer KJ, Ogbe A, Pace M, Adele S, Adland E, Alagaratnam J, Aley PK, Ali M, Ansari MA (2021) Safety and immunogenicity of the ChAdOx1 nCoV-19 (AZD1222) vaccine against SARS-CoV-2 in HIV infection: a single-arm substudy of a phase 2/3 clinical trial. Lancet HIV

59. Shinde V, Bhikha S, Hoosain Z, Archary M, Bhorat Q, Fairlie L, Lalloo U, Masilela MSL, Moodley D, Hanley S. Efficacy of NVX-CoV2373 Covid-19 vaccine against the B. 1.351 Variant. N Engl J Med. 2021;384:1899-909.

60. Buchbinder SP, Mehrotra DV, Duerr A, Fitzgerald DW, Mogg R, Li D, Gilbert PB, Lama JR, Marmor M, Del Rio C. Efficacy assessment of a cell-mediated immunity HIV-1 vaccine (the Step Study): a double-blind, randomised, placebo-controlled, test-ofconcept trial. Lancet. 2008;372:1881-93.

61. Zhu F-C, Li Y-H, Guan X-H, Hou L-H, Wang W-J, Li J-X, Wu S-P, Wang B-S, Wang Z, Wang L. Safety, tolerability, and immunogenicity of a recombinant adenovirus type- 5 vectored COVID19 vaccine: a dose-escalation, open-label, non-randomised, firstin-human trial. Lancet. 2020;395:1845-54.

62. Buchbinder SP, McElrath MJ, Dieffenbach C, Corey L. Use of adenovirus type-5 vectored vaccines: a cautionary tale. Lancet. 2020;396:e68-9.

63. Winwood JJ, Fitzgerald L, Gardiner B, Hannan K, Howard C, Mutch A. Exploring the social impacts of the COVID-19 pandemic on people living with HIV (PLHIV): a scoping review. AIDS Behav. 2021. https://doi.org/10.1007/s10461-021-03300-1.

64. Joint UN Programme on HIV/AIDS (UNAIDS) (2021) Statement of the Joint UN Programme on HIV/AIDS (UNAIDS) Interagency Working Group on Key Populations on the need to ensure access to quality, safe, and non-discriminatory services for HIV key populations and migrants in the context of the COVID-19 pandem.

65. Shiau S, Krause KD, Valera P, Swaminathan S, Halkitis PN. The burden of COVID-19 in people living with HIV: a syndemic perspective. AIDS Behav. 2020;24:2244-9.

66. Algarin AB, Varas-Rodríguez E, Valdivia C, Fennie KP, Larkey L, Hu N, Ibañez GE. Symptoms, stress, and HIV-related care among 
older people living with HIV during the COVID-19 pandemic, Miami, Florida. AIDS Behav. 2020;24:2236-8.

67. Santos G-M, Ackerman B, Rao A, et al. Economic, mental health, HIV prevention and HIV treatment impacts of COVID-19 and the COVID-19 response on a global sample of cisgender gay men and other men who have sex with men. AIDS Behav. 2021;25:311-21.

68. Jones DL, Morgan KE, Martinez PC, Rodriguez VJ, Vazquez A, Raccamarich PD, Alcaide ML. COVID-19 burden and risk among people with HIV. J Acquir Immune Defic Syndr. 2021;87:869-74.

69. Tomar A, Spadine MN, Graves-Boswell T, Wigfall LT. COVID19 among LGBTQ+ individuals living with HIV/AIDS: psycho-social challenges and care options. AIMS Public Heal. 2021;8:303-8.

70. Sanchez TH, Zlotorzynska M, Rai M, Baral SD. Characterizing the impact of COVID-19 on men who have sex with men across the United States in April, 2020. AIDS Behav. 2020;24:2024-32.

71. Stephenson R, Chavanduka TMD, Rosso MT, Sullivan SP, Pitter RA, Hunter AS, Rogers E. Sex in the time of COVID-19: results of an online survey of gay, bisexual and other men who have sex with men's experience of sex and HIV prevention during the US COVID-19 epidemic. AIDS Behav. 2021;25:40-8.

72. Hochstatter KR, Akhtar WZ, Dietz S, et al. Potential influences of the COVID-19 pandemic on drug use and HIV care among people living with HIV and substance use disorders: experience from a pilot mHealth intervention. AIDS Behav. 2021;25:354-9.

73. Dorward J, Khubone T, Gate K, et al. The impact of the COVID-19 lockdown on HIV care in 65 South African primary care clinics: an interrupted time series analysis. Lancet HIV. 2021;8:e158-65.
74. Ridgway JP, Schmitt J, Friedman E, Taylor M, Devlin S, McNulty M, Pitrak D. HIV care continuum and COVID-19 outcomes among people living with HIV during the COVID-19 pandemic, Chicago, IL. AIDS Behav. 2020;24:2770-2.

75. Adadi P, Kanwugu ON. Living with HIV in the time of COVID19: a glimpse of hope. J Med Virol. 2021;93:59-60.

76. Kowalska JD, Skrzat-Klapaczyńska A, Bursa D, et al. HIV care in times of the COVID-19 crisis - where are we now in Central and Eastern Europe? Int J Infect Dis. 2020;96:311-4.

77. Simões D, Stengaard AR, Combs L, Raben D. Impact of the COVID-19 pandemic on testing services for HIV, viral hepatitis and sexually transmitted infections in the WHO European Region, March to August 2020. Eurosurveillance. 2020;25:2001943.

78. Castel AD, Wilbourn B, Magnus M, Greenberg AE. SARS-CoV-2 and HIV: epidemiology, treatment, and lessons learned from HIV. AIDS Rev. 2020;22:133-42.

79. Marhefka S, Lockhart E, Turner D. Achieve research continuity during social distancing by rapidly implementing individual and group videoconferencing with participants: key considerations, best practices, and protocols. AIDS Behav. 2020;24:1983-9.

Publisher's Note Springer Nature remains neutral with regard to jurisdictional claims in published maps and institutional affiliations. 